\title{
Microaneurysm turnover in the macula is a biomarker for development of clinically significant macular edema in type 2 diabetes
}

This article was published in the following Dove Press journal:

Current Biomarker Findings

16 January 2013

Number of times this article has been viewed

\section{Luisa Ribeiro \\ Sandrina Nunes \\ José Cunha-Vaz}

Association for Innovation and Biomedical Research on Light and Image and Faculty of Medicine, University of Coimbra, Coimbra, Portugal
Correspondence: José Cunha-Vaz Association for Innovation and Biomedical Research on Light and Image, Azinhaga Santa Comba, Celas, 3000-548 Coimbra, Portugal Tel +35 I 239480 I36 $\mathrm{Fax}+35$ I 239480 । I7

Email cunhavaz@aibili.pt
Abstract: The evolution and progression of diabetic retinopathy varies between individuals and does not necessarily progress to vision loss in every patient. However, it is difficult in clinical practice to predict the clinical course and to identify which eyes will develop visionthreatening complications, ie, clinically significant macular edema or proliferative retinopathy. There is a clear need to identify biomarkers of disease progression. Microaneurysm turnover computed automatically in digital color fundus photography images using the RetmarkerDR is a good biomarker for worsening of retinopathy and development of clinically significant macular edema. For long-term prediction (ten years), a microaneurysm formation rate higher than two per year predicts development of clinically significant macular edema. For short-term prediction ( 2 years), a microaneurysm turnover rate lower than nine indicates that development of clinically significant macular edema is highly unlikely.

Keywords: biomarker, diabetes type 2, diabetic retinopathy, microaneurysms, retina

\section{Introduction}

Diabetic retinopathy is a chronic retinal disorder that affects nearly all patients with diabetes mellitus, albeit with different severity. Diabetic retinopathy is characterized by gradually progressive alterations in the retinal microvasculature, and is the leading cause of new cases of legal blindness among Americans aged 20-74 years. ${ }^{1}$ It is apparent from available data in a variety of large longitudinal studies and from clinical experience that the evolution and progression of diabetic retinopathy varies between individuals and does not necessarily progress to vision loss in every patient. Pivotal studies have confirmed the importance of metabolic control of diabetes in the prevention, development, and progression of diabetic retinopathy. ${ }^{2,3}$ Furthermore, treatments are now available that, together with laser photocoagulation, have been shown to be effective in delaying loss of vision.

However, it can be difficult in clinical practice to predict the clinical course and to identify which patients will develop vision-threatening complications of retinopathy. Duration of diabetes and control of glycemia and blood pressure only partially explain the variance in development and progression of diabetic retinopathy. ${ }^{3,4}$ For instance, patients with good glycemic control may develop rapidly progressive forms of diabetic retinopathy, while other people with relatively poor glycemic control may take several years to manifest signs of the disease. This discrepancy highlights the need to identify biomarkers of disease progression and the importance of a better understanding of the pathogenesis of diabetic retinopathy involved in disease progression for each individual. 
Biomarkers are indicators of biological and pathological processes that can be measured objectively. They are clinically useful when the biomarker precedes the development of dreaded outcomes (thus serving as a risk factor), or when the biomarker acts as a surrogate for an outcome that should be avoided. Identification of biomarkers for progression of diabetic retinopathy is expected to contribute to improved clinical monitoring and better risk stratification among patients with diabetes. Importantly, biomarkers should be an objective measure of disease progression that can be acquired quickly and be resource-saving without much strain on the patient. Here we review the available data on microaneurysm turnover as a retinal biomarker for progression of diabetic retinopathy to clinically significant macular edema, a major vision-threatening complication of diabetic retinopathy.

Microaneurysms and hemorrhages identified as red dots are the initial changes seen on ophthalmoscopic examination. They may be counted on fundus photography, and red dot counting has been suggested as an appropriate marker of retinopathy progression. ${ }^{5,6}$ It must be realized that red dot formation and disappearance are dynamic processes. During a 2-year follow-up of 24 type 1 diabetics with mild background diabetic retinopathy using fluorescein angiography, Hellstedt and Immonen observed 395 new microaneurysms and disappearance of 258 previously identified microaneurysms. ${ }^{7}$ Formation and disappearance of microaneurysms has been similarly identified in patients with type 2 diabetes. ${ }^{8,9}$

Generally, the disappearance of a microaneurysm is not a reversible process and indicates vessel closure and progressive vascular damage. Therefore, to assess progression of retinopathy, microaneurysm counting should take into account not only newly developed microaneurysms identified in a new location but also disappearing ones.

Microaneurysm disappearance is most likely because of thrombotic phenomena leading to subsequent rerouting of capillary blood flow and progressive remodeling of the retinal vasculature in diabetes. ${ }^{10}$ Therefore, the presence and number of microaneurysms and their rates of formation and disappearance are good candidates as biomarkers of retinal vascular remodeling and may be good indicators of progression of retinopathy.

\section{RetmarkerDR software}

Counting of microaneurysms on color fundus photography and fluorescein angiography have been proposed as predictive indicators for progression of diabetic retinopathy. ${ }^{11}$ Recently developed software, the RetmarkerDR (Critical Health SA, Coimbra, Portugal), allows identification of the exact location of each red dot in successive fundus photographs performed in each eye. Identification of the exact location of an individual red dot is considered particularly important, because a new microaneurysm is considered to develop only once in a specific location, its disappearance generally being associated with capillary closure, leaving in its place mainly remnants of basement membrane. ${ }^{12,13}$

The automated computer-aided diagnostic system RetmarkerDR consists of software marking microaneurysms and vascular lesions, and includes a coregistration algorithm that allows comparison within the same retinal location between different visits for the same eye. Being a deterministic algorithm, its performance is not affected by fatigue, stress, ambient light conditions, or any other factors that may influence human grading. Thus, the RetmarkerDR brings objectivity to subjective tasks (Figures 1 and 2).

The algorithm detects the presence of microaneurysms and red dot-like lesions. Because microaneurysms are identified by ophthalmoscopy or color fundus photography as deep red dots, sometimes they are difficult to differentiate from punctate hemorrhages or localized vascular abnormalities. Punctate hemorrhages typically have irregular borders and may be wrongly classified as microaneurysms if the borders happen to be more regular. ${ }^{14-17}$

In order to assess disease activity in the central $3000 \mu \mathrm{m}$ circle of the macula, images from field 2 (macula) are automatically coregistered. ${ }^{18}$ Image coregistration is achieved by extracting the retinal vascular tree, which is used for landmarks during the registration process. A rigid registration estimates the translation based on fovea displacement. The rotation is estimated using a polar representation of the vascular tree. This rigid transformation is then adjusted to obtain exact pairings of selected landmarks. ${ }^{19}$ In this way, it is possible to classify each microaneurysm detected as being new (not present in the previous visit), old (present in the previous visit), or disappeared (no longer present).

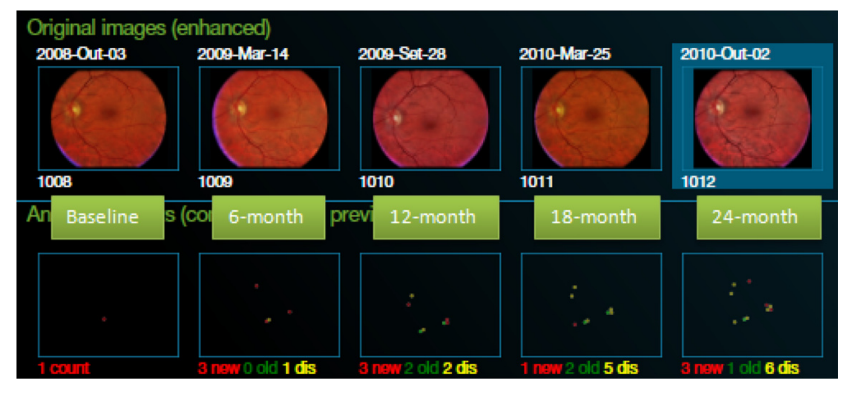

Figure I Automatic microaneurysm tracking over time, color coding detecting each microaneurysm as new, old, or disappeared. 


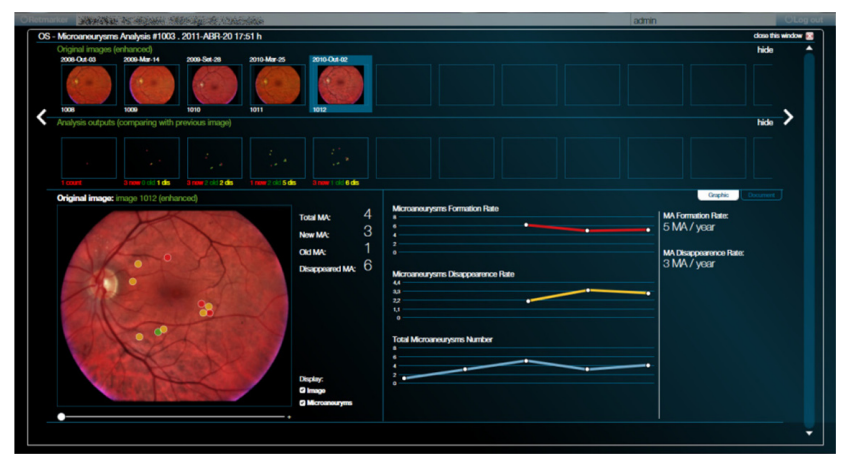

Figure 2 The RetmarkerDR software automatically calculates microaneurysm formation and disappearance rates.

Notes: The patient above had a microaneurysm formation rate of five microaneurysms per year over a 24-month follow-up.

The RetmarkerDR allows the number of microaneurysms at each visit to be computed for each eye, along with the number of new microaneurysms that appear between visits over a period of time (microaneurysm formation rate), number of microaneurysms that disappear between visits over a period of time (microaneurysm disappearance rate), and turnover, ie, the sum of microaneurysm formation and disappearance rates.

\section{Results}

Our studies have demonstrated a steady turnover of microaneurysms in the diabetic retina, even in the initial stages of retinopathy. In fact, most microaneurysms have a lifetime of less than one year, with new ones being formed and disappearing at rates which vary between different patients, confirming previous reports. ${ }^{20}$

Using the RetmarkerDR, we analyzed data from a group of 113 type 2 diabetic patients with mild to moderate nonproliferative diabetic retinopathy, followed up for 2 years as controls in diabetic retinopathy clinical trials, and thereafter by usual care at the same institution. ${ }^{14}$

Microaneurysm turnover from the initial 2 years and the occurrence of clinically significant macular edema during the following 8 years were analyzed in this retrospective 10 -year follow-up study. At the end of 10-year follow-up, 17 of the 113 patients had developed clinically significant macular edema needing photocoagulation. At baseline, patients who developed clinically significant macular edema had mean $\mathrm{HbA}_{1 \mathrm{C}}$ levels that were significantly higher $(8.5 \% \pm 1.2 \%)$ than in the patients who did not $(7.3 \% \pm 1.2 \%, P=0.001)$. No statistically significant differences were found between clinically and nonclinically significant macular edema for blood pressure, cholesterol, high-density lipoprotein, lowdensity lipoprotein, and triglyceride levels at baseline.
When counting the total number of microaneurysms over the first 2 years of follow-up, a significant increase in the number of microaneurysms was found for eyes with clinically significant macular edema $(P=0.002)$, while the number of microaneurysms remained relatively constant for eyes with nonclinically significant macular edema $(P=0.647)$.

When computing the microaneurysm turnover rate, significantly higher values were found in the group of eyes that developed clinically significant macular edema. Mean formation rates of $9.2 \pm 18.2$ microaneurysms per year were found for eyes that developed clinically significant macular edema, while mean formation rates of $0.5 \pm 1.2$ microaneurysms per year were found for eyes that did not $(P<0.001)$. A microaneurysm formation rate of at least two microaneurysms per year was found in 12 of the 17 eyes that developed clinically significant macular edema $(70.6 \%)$, whereas this was only found in eight of the 96 eyes $(8.3 \%)$ that did not during the 10-year follow-up period. This study showed that higher microaneurysm turnover obtained from color fundus photography in the initial stages of diabetic retinopathy is a good indicator of retinopathy activity and a predictor of development of clinically significant macular edema needing photocoagulation.

Our results have since been confirmed by another research group in Munich also using the RetmarkerDR (Ulbig et al, data presented at 11th EURETINA congress, London, UK, May 26-29, 2011). ${ }^{21}$ In their study, they retrospectively analyzed a group of 160 eyes followed by fundus photography for a period of 5 years in the CALDIRET study, ${ }^{22}$ and were able to compare 49 eyes that did develop clinically significant macular edema over the period of the study with 111 eyes that did not. An increased microaneurysm formation rate was confirmed to be clearly associated with development of clinically significant macular edema. Values indicating a microaneurysm formation rate of greater than two per year in this early stage of retinopathy were present in $71.4 \%$ of eyes that developed clinically significant macular edema. In marked contrast, the eyes that did not develop this complication during the study showed a microaneurysm formation rate of less than two per year in $68.5 \%$ of cases. This study, which also used the RetmarkerDR software, confirms the research done by our group, in which $70.6 \%$ of eyes that developed clinically significant macular edema showed a microaneurysm formation rate greater than two.

\section{Discussion}

Sharp et al found that the microaneurysm turnover rate varied widely between eyes with the same retinopathy level. ${ }^{23}$ 
This is also consistent with our findings. Microaneurysm turnover was shown in our study to vary widely between patients who were classified with the same retinopathy level. Microaneurysm turnover computed from noninvasive color fundus photography appears to be a good biomarker to identify patients at risk of progression to clinically significant macular edema and may serve as a surrogate outcome for its development.

More recently, we performed a prospective observational study designed to follow patients with mild nonproliferative diabetic retinopathy (grades 20 and 35) for a period of 2 years or until the time of development of a vision-threatening complication of diabetic retinopathy, ie, clinically significant macular edema needing laser photocoagulation. ${ }^{17} \mathrm{~A}$ total of 410 patients with diagnosed adult-onset type 2 diabetes, including both men and women aged 40-75 years with mild nonproliferative diabetic retinopathy (levels 20 and 35 of Early Treatment Diabetic Retinopathy Study [ETDRS] classification) and best-corrected visual acuity $\geq 95$ ETDRS letters (20/25). One eye per patient was selected as the study eye. At the three study visits, ie, at entry, 6 months, and 24 months, the study eye underwent a complete examination which included fundus photography and optical coherence tomography. The field 2 color fundus images were subjected to automated microaneurysm analysis using the RetmarkerDR. Of the 410 eyes entered into the study, 348 were considered for analysis because they reached either the study endpoint (clinically significant macular edema needing laser photocoagulation) or attended the last visit at 24 months. Of these 348 eyes, 26 were diagnosed during the 2-year period of follow-up as having clinically significant macular edema and were treated with laser photocoagulation. The other 322 eyes from patients who completed the last follow-up visit at 24 months did not develop clinically significant macular edema.

Microaneurysm turnover calculated at the 6-month follow-up was $11.2 \pm 11.2$ in the 26 eyes that developed clinically significant macular edema and 5.0 \pm 5.2 in the remaining 322 eyes $(P<0.001)$. For a microaneurysm turnover cutoff of nine or more, a sensitivity of $57.7 \%$ and a specificity of $81.2 \%$ was achieved (ie, $79.4 \%$ of the eyes were correctly classified). The predictive values for microaneurysm turnover for clinically significant macular edema development for the 2 years of follow-up were $20 \%$ for the positive predictive value and $96 \%$ for the negative predictive value, showing that a low microaneurysm turnover value predicts slow disease progression and indicates that development of clinically significant macular edema is unlikely.
When considering systemic parameters, only $\mathrm{HbA}_{1 \mathrm{c}}$ values at baseline correlated with development of clinically significant macular edema. The other systemic parameters examined, including systolic and diastolic blood pressure and blood lipids (triglycerides, cholesterol, high-density lipoprotein, and low-density lipoprotein) did not show any correlation with occurrence of clinically significant macular edema. Multivariate analysis also showed that microaneurysm turnover is predictive of clinically significant macular edema independently of $\mathrm{HbA}_{1 \mathrm{c}}$ levels.

This 2-year, prospective, longitudinal study of patients with diabetes type 2 and mild nonproliferative diabetic retinopathy (ETDRS levels 20 and 35) showed that microaneurysm turnover in field 2 of the eye fundus is a good biomarker for worsening of retinopathy and development of clinically significant macular edema needing photocoagulation.

\section{Conclusion}

These results, based on precise identification of the location of each red dot on color fundus photographs of diabetic eyes, suggest that microaneurysm formation and disappearance rates may be an appropriate retinal biomarker of disease activity and progression of retinopathy to clinically significant macular edema, a clinical outcome that is associated with vision loss.

From the available data, for long-term prediction (10 years), a microaneurysm formation rate higher than two per year predicts the development of clinically significant macular edema. For short-term prediction (2 years), a microaneurysm turnover lower than nine indicates that development of clinically significant macular edema is highly unlikely.

Digital fundus imaging is ideally suited to quality assurance and will allow more efficient utilization in widespread screening programs. Automated analysis techniques offer the advantages of repeatability and consistency, and, although not necessarily better than individual graders or ophthalmologists in absolute terms, they avoid the variability inherent between individual human graders who have their own varying internal reference standards.

Our studies show that microaneurysm turnover obtained from field 2 color fundus photographs can predict the development of a vision-threatening complication of diabetic retinopathy in the early stages of nonproliferative diabetic retinopathy, ie, clinically significant macular edema, both in the short term and long term. 


\section{Disclosure}

The authors report no conflicts of interest in this work.

\section{References}

1. Aiello LP, Gardner TW, King GL, et al. Diabetic retinopathy. Diabetes Care. 1998;21:143-156.

2. Diabetes Control and Complications Trial Research Group. The effect of intensive treatment on of diabetes on the development and progression of long-term complications in insulin-dependent diabetes mellitus. N Engl J Med. 1993;329:977-986.

3. Holman RR, Paul SK, Bethel MA, Matthews DR, Neil HA. 10-year follow-up of intensive glucose control in type 2 diabetes. $N$ Engl J Med. 2008;359:1577-1589.

4. Hove MN, Kristensen JK, Lauritzen T, Bek T. The relationships between risk factors and the distribution of retinopathy lesions in type 2 diabetes. Acta Ophthalmol Scand. 2006;84:619-623.

5. Klein R, Klein BEK, Moss SE, Cruickshanks KJ. The Wisconsin Epidemiologic Study of Diabetic Retinopathy. XV. The long-term incidence of macular edema. Ophthalmology. 1995;102:7-16.

6. Klein R, Meuer SM, Moss SE, Klein BEK. Retinal microaneurysms counts and 10-year progression of diabetic retinopathy. Arch Ophthalmol. 1995;113:1386-1391.

7. Hellstedt T, Immonen I. Disappearance and formation rates of microaneurysms in early diabetic retinopathy. $\mathrm{Br} J$ Ophthalmol. 1996;80: 135-139.

8. Hohner EM, Stratton IM, Aldington SJ, Turner RC, Matthews DR; for the UK Prospective Diabetes Study (UKPDS) Group. Microaneurysms in the development of diabetic retinopathy (UKPDS 42). Diabetologia. 1999;42:1107-1112.

9. Sjolie AK, Klein R, Orchard T, et al. Retinal microaneurysm count predicts progression and regression of diabetic retinopathy. Post-hoc results from the DIRECT Programme. Diabet Med. 2011; 28:1-7.

10. Boeri D, Maiello M, Lorenzi M. Increased prevalence of microthromboses in retinal capillaries of diabetic individuals. Diabetes. 2001;50: 1432-1439.

11. Kohner EM, Sleightholm M, The KROC collaborative study group. Does microaneurysm count reflect severity of early diabetic retinopathy? Ophthalmology. 1986;93:586-589.
12. Ashton N. Vascular basement membrane changes in diabetic retinopathy. Montgomery Lecture, 1973. Br J Ophthalmol. 1974;58:344-347.

13. Cunha-Vaz JG. Pathophysiology of diabetic retinopathy. $\mathrm{Br} J$ Ophthalmol. 1978;62:351-355.

14. Nunes S, Pires I, Rosa A, Duarte L, Bernardes R, Cunha-Vaz J. Microaneurysm turnover is a biomarker for diabetic retinopathy progression to clinically significant macular edema: findings for type 2 diabetics with nonproliferative retinopathy. Ophthalmologica. 2009;223: 292-297.

15. Fleming AD. Automated microaneurysm detection using local contrast normalization and local vessel detection. IEEE Trans Med Imaging. 2006;9:1223-1232.

16. Tuzel O, Porikli F, Meer P. Region covariance. A fast descriptor for detection and classification. In: Leonardis A, Bischof H, Pinz A, editors. Computer Vision - ECCV 2006. Berlin, Germany: Springer; 2006.

17. Ribeiro ML, Nunes S, Cunha-Vaz J. Microaneurysm turnover at the macula predicts risk of development of clinically significant macular edema in persons with mild non-proliferative diabetic retinopathy. Diabetes Care. November 30, 2012. [Epub ahead of print.]

18. Bernardes R, Baptista P, Cunha-Vaz J, Dias J. Multimodal functional and morphological nonrigid image registration. Presented at the 2005 International Conference on Image Processing, Genoa, Italy, September 11-14, 2005.

19. Ferreira J, Bernardes R, Baptista P, Dias J, Cunha-Vaz J. Earmarking retinal changes in a sequence of digital color fundus photographs. Presented at the 3rd European Medical and Biological Engineering Conference, Prague, Czech Republic, November 20-25, 2005.

20. Kohner EM, Dollery CT. The rate formation and disappearance of microaneurysms in diabetic retinopathy. Eur J Clin Invest. 1970;1: $167-171$.

21. Ulbig M. Fundus photography as a marker for diabetic macular edema. In: 11 th EURETINA CONGRESS: European vitreoretinal update 2011; May 26-29, 2011; London, UK; 2011;9:2.

22. Christos H, Gerss J, Sauerland C, Kampik A, Ulbig MW; for the CALDIRET Study Group. Effect of calcium dobesilate on occurrence of diabetic macular oedema (CALDIRET study): randomised, double-blind, placebo-controlled, multicentre trial. Lancet. 2009;373: 1364-1371.

23. Sharp PF, Olson J, Strachan F, et al. The value of digital imaging in diabetic retinopathy. Health Technol Assess. 2003;7:1-119.
Current Biomarker Findings

\section{Publish your work in this journal}

Current Biomarker Findings is an international, peer-reviewed, open access journal publishing original research, reports, reviews and commentaries on all areas of biomarker research. The manuscript management system is completely online and includes a very quick and fair

\section{Dovepress}

peer-review system. Visit http://www.dovepress.com/testimonials.php to read real quotes from published authors. 